Quim. Nova, Vol. 35, No. 8, 1554-1559, 2012

\title{
GERAÇÃO DE MESOPOROS EM ZEÓLITAS ZSM-5 E SEUS EFEITOS NA CONVERSÃO DO ETANOL EM OLEFINAS
}

\author{
Ingryd Carvalho Alves, Tâmara Lucia Pacheco de Mattos do Nascimento\#, Cláudia de Oliveira Veloso, Fátima Maria Zanon \\ Zotin e Cristiane Assumpção Henriques* \\ Instituto de Química, Universidade do Estado do Rio de Janeiro, Rua São Francisco Xavier, 524, 20550-900 Rio de Janeiro - RJ, \\ Brasil
}

Recebido em 9/12/11; aceito em 5/4/12; publicado na web em 3/7/12

\begin{abstract}
MESOPORE GENERATION IN ZSM-5 ZEOLITES AND THEIR EFFECTS ON CONVERSION OF ETHANOL TO OLEFINS. Developing mesoporosity in HZSM-5 zeolites is an alternative for improving their catalytic performance on chemical reactions. In this work, alkaline and thermal treatments were used to produce mesoporosity. These treatments increased mesoporous area and volume. They also influenced the acid properties of the samples. Concerning catalytic performance, treatments modified reaction product distribution. Whereas alkaline treatment favored formation of olefins and increased propene ratio in the beginning of the reaction, thermal treatment resulted in formation of only ethylene due to the low acidity of the sample.
\end{abstract}

Keywords: ethanol; mesopores generation; ZSM-5 zeolite.

\section{INTRODUÇÃO}

A produção a partir de fontes de biomassa renovável e a possibilidade de obtenção de hidrocarbonetos como propeno, butenos e BTXE (benzeno, tolueno, xilenos e etilbenzeno) são características do etanol, que impulsionam a busca por processos catalíticos que possibilitem sua utilização. A crescente demanda por estes hidrocarbonetos, a instabilidade no fornecimento e nos preços dos combustíveis fósseis e o aumento das emissões de $\mathrm{CO}_{2}$ também colaboram para o crescente interesse nas reações de transformação do etanol.

O propeno é uma importante matéria-prima para a síntese de produtos químicos como óxido de propileno, polipropileno, acroleína e ácido acrílico, sendo principalmente produzido pelo craqueamento térmico da nafta. $\mathrm{O}$ desenvolvimento de novos processos para a obtenção de propeno a partir de etanol diminuiria a dependência de fontes fósseis e as emissões de $\mathrm{CO}_{2}$.

A seletividade de forma e a força ácida moderada da zeólita HZSM-5 são adequadas à transformação de bioetanol em eteno, propeno, butenos ou hidrocarbonetos superiores. Foram testadas zeólitas HZSM-5 com diferentes relações sílica/alumina (SAR) modificadas ou não com metais para a produção seletiva de propeno..$^{1-4}$ Song e colaboradores ${ }^{1}$ estudaram zeólitas ZSM-5 com SAR (razão molar $\mathrm{SiO}_{2} / \mathrm{Al}_{2} \mathrm{O}_{3}$ ) igual a 30,80 e 280 e verificaram que a zeólita HZSM-5 com SAR 80 proporcionou o maior rendimento em propeno, indicando que uma acidez moderada favorece a produção de propeno. Também verificaram que a introdução de zircônio por impregnação úmida numa relação de $\mathrm{Zr} / \mathrm{Al}=0,4^{1}$ ou a incorporação de fósforo na proporção de $\mathrm{P} / \mathrm{Al}=0,5^{4}$ em uma zeólita H-ZSM-5 (SAR 80) proporcionou um rendimento em propeno de $32 \%$ e uma melhora na estabilidade catalítica da zeólita.

Alguns fatores são fundamentais para o desenvolvimento de processos viáveis para a transformação de etanol em hidrocarbonetos. São eles, a estabilidade hidrotérmica, a seletividade do catalisador zeolítico e a minimização da desativação por formação de coque. A produção de olefinas $\mathrm{C}_{3}-\mathrm{C}_{4}$ e aromáticos $\mathrm{BTXE}$ requer temperaturas superiores a $300{ }^{\circ} \mathrm{C}$, que aumentam a taxa de desativação

*e-mail: cah@uerj.br

\#Endereço atual: HPE/CENPES/PETROBRAS, Rio de Janeiro, RJ - Brasil por formação de coque. ${ }^{5}$ Madeira e colaboradores ${ }^{6}$ provaram que a formação de coque ocorre principalmente nos microporos, que são bloqueados por intermediários alquil aromáticos identificados por espectroscopia na região do infravermelho. Gayubo e colaboradores ${ }^{7}$ trataram uma zeólita HZSM-5 (SAR 30) com uma solução diluída de $\mathrm{NaOH}$ por um curto tempo e observaram que este tratamento foi efetivo para minimizar a desativação por deposição de coque. $\mathrm{O}$ pequeno aumento da mesoporosidade, que reduziu o bloqueio dos poros pela formação de coque, e o decréscimo da acidez, que inibiu as etapas de crescimento do coque, foram responsáveis pela menor desativação.

A geração de mesoporosidade em zeólitas pode ser realizada por diferentes rotas, ${ }^{8}$ tais como a síntese direta do aluminossilicato mesoporoso, a utilização de direcionadores de carbono no gel de síntese e as modificações pós-síntese, como os tratamentos hidrotérmico, térmico, alcalinos ou ácidos. A criação de mesoporos através de tratamento térmico pós-síntese foi estudada por Zhang e colaboradores ${ }^{9}$ para zeólitas ZSM-5, sendo considerado um método de baixo custo, fácil controle e boa reprodutibilidade para a obtenção de materiais bimodais. No entanto, foi observada uma destruição parcial da estrutura cristalina. Já o uso do tratamento alcalino remove átomos de silício da estrutura cristalina da zeólita (dessilicação) gerando mesoporos e preservando os átomos de $\mathrm{Al}$ associados à acidez do material. ${ }^{10}$ Neste método, a concentração da solução de $\mathrm{NaOH}$ é fundamental para a formação de mesoporos sem perda de cristalinidade da zeólita. ${ }^{11}$

Neste contexto, o presente trabalho estudou o desempenho catalítico de amostras de zeólita HZSM-5 (SAR 27) modificadas através de tratamento alcalino com solução diluída de $\mathrm{NaOH}$ e de tratamento térmico a altas temperaturas perante a transformação de etanol em hidrocarbonetos, especialmente propeno.

\section{PARTE EXPERIMENTAL}

\section{Preparo dos catalisadores}

Uma zeólita ZSM-5, na forma amoniacal com SAR nominal igual a 27,3 cedida pelo CENPES/PETROBRAS, foi utilizada como material de partida para os tratamentos alcalino e térmico. A forma 
ácida da amostra (HZSM-5) foi obtida por calcinação em atmosfera de ar estático a $450{ }^{\circ} \mathrm{C}$ por $4,5 \mathrm{~h}$.

O tratamento térmico foi realizado de acordo com as condições estabelecidas por Nascimento. ${ }^{12}$ A zeólita na forma amoniacal foi aquecida em mufla em atmosfera de ar estático. A temperatura final de tratamento foi alcançada de acordo com a seguinte programação: aquecimento até $300{ }^{\circ} \mathrm{C}$ com taxa de $30{ }^{\circ} \mathrm{C} \mathrm{min}^{-1}$ mantendo-se nesta temperatura por $30 \mathrm{~min}$, seguido de aquecimento até $1000{ }^{\circ} \mathrm{C}$ com taxa de $70{ }^{\circ} \mathrm{C} \mathrm{min}{ }^{-1}$ mantendo-se nesta temperatura por $2 \mathrm{~h}$. A amostra foi denominada TZSM-5.

O tratamento básico foi realizado com solução de $\mathrm{NaOH}$ (Vetec, $97 \%$ P.A.) a $0,2 \mathrm{~mol} \mathrm{~L}^{-1}$ usando uma razão massa de zeólita na forma amoniacal $(\mathrm{g}) /$ volume de solução de $\mathrm{NaOH}(\mathrm{mL})$ igual a 0,008 . A suspensão foi agitada mecanicamente a $85^{\circ} \mathrm{C}$ por $2 \mathrm{~h}$. Em seguida, a suspensão foi resfriada rapidamente até a temperatura ambiente, filtrada, lavada com água desmineralizada até $\mathrm{pH}$ neutro, e seca a $150{ }^{\circ} \mathrm{C}$ por $1 \mathrm{~h}$. Considerando que o tratamento com a solução de $\mathrm{NaOH}$ promove a substituição dos cátions de compensação $\mathrm{NH}_{4}^{+}$ por $\mathrm{Na}^{+}$, a amostra tratada foi submetida a uma troca iônica com solução de $\mathrm{NH}_{4} \mathrm{Cl}$ (Vetec, 99,8\%) a $2 \mathrm{~mol} \mathrm{~L}^{-1}$, visando a troca dos íons $\mathrm{Na}^{+}$por $\mathrm{NH}_{4}^{+}$, de modo a gerar posteriormente a forma ácida, por calcinação a $450{ }^{\circ} \mathrm{C}$ por $4,5 \mathrm{~h}$, em ar, sob atmosfera estática. A amostra foi denominada BZSM-5.

\section{Caracterização físico-química dos catalisadores}

Todos os catalisadores utilizados foram analisados por adsorção de nitrogênio a $-196{ }^{\circ} \mathrm{C}$ (Micromeritics-ASAP 2020) para determinação de suas propriedades texturais, tais como, área específica, volume de microporos, área e volume de mesoporos e por difração de raios X (difratômetro Rigaku, modelo Miniflex II), utilizando-se radiação $\mathrm{CuK} \alpha$ com voltagem de $30 \mathrm{kV}$ e corrente de $15 \mathrm{~mA}$, para a determinação da cristalinidade, calculada a partir das intensidades dos picos referentes aos planos (501), (303) e (133).

A determinação da SAR da rede cristalina bem como o percentual de alumínio extrarrede das zeólitas estudadas foi realizada através da espectroscopia de ressonância magnética nuclear com rotação no ângulo mágico (MAS/RMN) do ${ }^{29} \mathrm{Si}$ e do ${ }^{27} \mathrm{Al}$. O instrumento utilizado (Varian Infinity-Plus-400) foi equipado com sondas VT CP/MAS de 7,5 mm específicas para amostras sólidas e utilizadas para observação dos elementos desejados. Os espectros de MAS/RMN do ${ }^{29} \mathrm{Si}$ foram obtidos a 79,2 MHz, empregando-se pulsos de $1,0 \mu \mathrm{s}(\pi / 2)$ e $20 \mathrm{~s}$ de intervalo entre os pulsos, sendo acumulados um total de 500 pulsos. A rotação no ângulo mágico foi $5 \mathrm{MHz}$. Como referência foi utilizado o caulim $(\delta=-91,5 \mathrm{ppm})$. Os espectros de MAS/RMN do ${ }^{27} \mathrm{Al}$ foram obtidos a $103,9 \mathrm{MHz}$, empregando-se pulsos de $1,0 \mu \mathrm{s}(\pi / 20)$ e $0,5 \mathrm{~s}$ de intervalo entre os pulsos, sendo acumulados um total de 5000 pulsos. A rotação no ângulo mágico foi $10 \mathrm{MHz}$ e os espectros foram referenciados ao $\mathrm{AlCl}_{3} \cdot 6 \mathrm{H}_{2} \mathrm{O}(\delta=0 \mathrm{ppm})$.

A densidade e a distribuição de força dos sítios ácidos das amostras foram determinadas através da dessorção de $\mathrm{NH}_{3}$ à temperatura programada (TPD de $\mathrm{NH}_{3}$ ). Para tal, foi utilizado um sistema dinâmico, passando-se uma mistura gasosa contendo 2,91\% de $\mathrm{NH}_{3}$ em He por um reator tubular contendo a amostra. A composição da mistura gasosa na saída do reator foi determinada por um detector de condutividade térmica. Antes da adsorção do $\mathrm{NH}_{3}$, a amostra foi tratada a $150{ }^{\circ} \mathrm{C}$ por $1 \mathrm{~h}$ e, em seguida, a $500{ }^{\circ} \mathrm{C}$ por $1 \mathrm{~h}$, sob uma corrente de $30 \mathrm{~mL} \mathrm{~min}^{-1}$ de $\mathrm{He}$, sendo o aquecimento feito com uma taxa de $10{ }^{\circ} \mathrm{C} \mathrm{min}^{-1}$. A densidade de sítios ácidos foi calculada usando-se a quantidade de $\mathrm{NH}_{3}$ adsorvida quimicamente a $150{ }^{\circ} \mathrm{C}$, obtida pela diferença entre a quantidade total adsorvida e a adsorvida fisicamente.

\section{Testes catalíticos}

A conversão de etanol em hidrocarbonetos foi realizada em fase vapor, sendo o etanol (Tedia Brasil) alimentado através de um saturador, utilizando-se uma corrente de nitrogênio como gás de arraste. Os testes catalíticos foram realizados nas seguintes condições experimentais: pressão atmosférica; temperatura do saturador $=32{ }^{\circ} \mathrm{C}$ (pressão parcial de etanol $=0,11 \mathrm{~atm}$ ); gás de arraste $\left(\mathrm{N}_{2}\right)$ a uma vazão de $50 \mathrm{~mL} \mathrm{~min}^{-1}$; temperatura de reação $=500{ }^{\circ} \mathrm{C}$; massa de catalisador $=0,1000 \mathrm{~g}$; velocidade espacial (WHSV) igual a 6,5 $\mathrm{g}_{\text {EtOH }} \mathrm{h}^{-1} \mathrm{~g}_{\text {cat }}{ }^{-1}$. Os produtos de reação foram analisados em linha por cromatografia em fase gasosa empregando um cromatógrafo Varian 3900, munido de uma coluna capilar HP-Plot/Q de 30m e de um detector de ionização por chama.

Antes do teste catalítico, as amostras foram pré-tratadas in situ, nas seguintes condições: aquecimento sob corrente de $\mathrm{N}_{2}$ de $50 \mathrm{~mL}$ $\min ^{-1}$ a $150{ }^{\circ} \mathrm{C}$ por $1 \mathrm{~h} \mathrm{e}$, em seguida, a $500{ }^{\circ} \mathrm{C}$ também por $1 \mathrm{~h}$ com taxa de aquecimento de $10{ }^{\circ} \mathrm{C} \mathrm{min}^{-1}$.

\section{RESULTADOS E DISCUSSÃO}

O efeito dos tratamentos térmico e básico sobre as propriedades texturais das zeólitas preparadas pode ser observado na Tabela 1. Os dois procedimentos proporcionaram aumento tanto na área quanto no volume de mesoporos, sendo que o tratamento básico foi mais eficaz na geração de mesoporosidade. A formação de mesoporos também foi verificada através das isotermas de fisissorção de $\mathrm{N}_{2}$ das amostras tratadas, Figura 1, onde um nítido aumento no ciclo de histerese foi notado, assim como a mudança na classificação do tipo da isoterma. A amostra HZSM-5 apresentou uma isoterma do tipo I, característica de materiais microporosos, enquanto a amostra BZSM-5 apresentou isoterma do tipo IV. No caso da amostra TZSM-5, nota-se a presença incipiente de um ciclo de histerese, associado à baixa mesoporosidade gerada. Observa-se, ainda, que nos dois tratamentos o aumento da mesoporosidade foi acompanhado de redução na área e no volume de microporos, calculados pelo método t como ilustrado na Figura $1 \mathrm{~S}$, material suplementar, sugerindo que a formação dos mesoporos tenha ocorrido a partir do colapso parcial dos microporos promovido pelos tratamentos realizados.

Tabela 1. Propriedades texturais das amostras HZSM-5, TZSM-5 e BZSM-5

\begin{tabular}{lccc}
\hline Amostra & HZSM-5 & BZSM-5 & TZSM-5 \\
\hline $\mathrm{S}_{\text {BET }}\left(\mathrm{m}^{2} \mathrm{~g}^{-1}\right)$ & 370 & 343 & 341 \\
$\mathrm{~S}_{\text {micro }}{ }^{\mathrm{a}}\left(\mathrm{m}^{2} \mathrm{~g}^{-1}\right)$ & 363 & 206 & 308 \\
$\mathrm{~S}_{\text {meso+ext }}{ }^{\mathrm{a}}\left(\mathrm{m}^{2} \mathrm{~g}^{-1}\right)$ & 7 & 136 & 33 \\
$\mathrm{~V}_{\text {micro }}{ }^{\mathrm{a}}\left(\mathrm{m}^{3} \mathrm{~g}^{-1}\right)$ & 0,172 & 0,091 & 0,149 \\
$\mathrm{~V}_{\text {meso }}{ }^{\mathrm{b}}\left(\mathrm{m}^{3} \mathrm{~g}^{-1}\right)$ & 0,022 & 0,221 & 0,065 \\
\hline
\end{tabular}

a: calculado pelo método t-plot (5 -10 Å, Figura $1 \mathrm{~S}$, material suplementar); ${ }^{\mathrm{b}}$ : calculado pelo método BJH

A composição química (razão molar $\mathrm{Si} / \mathrm{Al}$ ) da rede cristalina das zeólitas estudadas, medida por MAS/RMN do ${ }^{29} \mathrm{Si}$, e a distribuição das espécies de alumínio presentes, medida por MAS/RMN do ${ }^{27} \mathrm{Al}$, são mostradas na Tabela 2, sendo os espectros correspondentes apresentados nas Figuras $2 \mathrm{~S}$ e $3 \mathrm{~S}$, respectivamente, material suplementar. Para as três amostras estudadas, os espectros de MAS/RMN do ${ }^{29} \mathrm{Si}$ apresentaram um pico intenso em aproximadamente $-115 \mathrm{ppm}$, correspondente aos átomos de silício ligados a quatro outros átomos de silício (4Si-0Al) através do átomo de oxigênio. ${ }^{13}$ No caso das amostras HZSM-5 e BZSM-5, foi observada a presença de um segundo pico a cerca de -109 ppm, atribuído aos átomos de silício ligados a três 


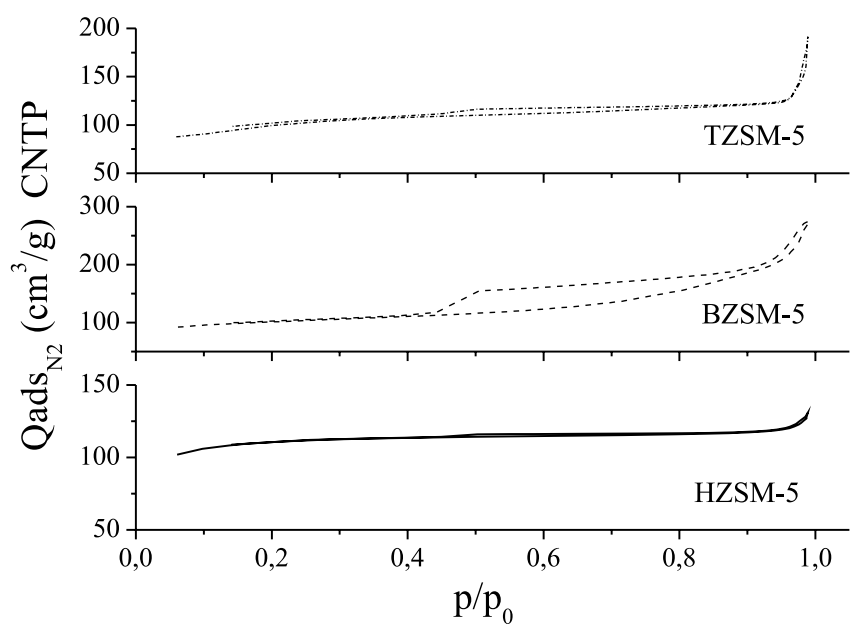

Figura 1. Isotermas de fisissorção do $N_{2}$ a $-196{ }^{\circ} \mathrm{C}$ das amostras HZSM-5, TZSM-5 e BZSM-5

Tabela 2. Percentual de cristalinidade, determinado por difratometria de raios X, razão molar Si/Al e distribuição das espécies de $\mathrm{Al}$ determinadas por espectroscopia de ressonância magnética nuclear com rotação no ângulo mágico do ${ }^{29} \mathrm{Si}$ e do ${ }^{27} \mathrm{Al}$

\begin{tabular}{lccc}
\hline Amostra & HZSM-5 & BZSM-5 & TZSM-5 \\
\hline Si/Al rede ${ }^{\text {a }}$ & 18,6 & 14,7 & n.d. $^{\text {b }}$ \\
\% de Al tetraédrico $^{c}$ & 92,3 & 66,5 & 39,8 \\
\% de Al extra rede $^{c}$ & 7,7 & 33,5 & 60,2 \\
\% cristalinidade & 100 & 84 & 76 \\
\hline
\end{tabular}

a: MAS/RMN do ${ }^{29} \mathrm{Si}$; ${ }^{\text {b: }}$ não determinado por esta técnica devido à intensa desaluminização; ${ }^{c}$ : MAS/RMN do ${ }^{27} \mathrm{Al}$

outros átomos de silício e a um átomo de alumínio (3Si-1Al), também através de átomo de oxigênio comum. ${ }^{13}$

Os espectros de MAS/RMN do ${ }^{27} \mathrm{Al}$ apresentaram dois picos principais. Um correspondente ao alumínio em coordenação tetraédrica, associado ao alumínio da rede cristalina da zeólita, a cerca de $55 \mathrm{ppm}$, e outro, com deslocamento em torno de $0,5 \mathrm{ppm}$, associado ao alumínio em coordenação octaédrica e correspondente às espécies extrarreticulares (ALER). No caso da amostra TZSM-5, foi observada também a presença de um pico largo, com deslocamento entre 20 e $45 \mathrm{ppm}$, e que pode ser atribuído a alumínio extrarrede em coordenação tetraédrica com constante quadrupolar muito elevada e/ou a alumínio pentacoordenado. ${ }^{13,14} \mathrm{Na}$ impossibilidade de realização de experimentos que permitissem identificá-los inequivocamente (análises pela técnica de MQMAS/RMN do ${ }^{27} \mathrm{Al}$ ), foram considerados como espécies de alumínio extrarrede (ALER).

A Tabela 2 mostra que, no caso da amostra HZSM-5, o valor da razão $\mathrm{Si} / \mathrm{Al}$ foi superior ao do precursor, sugerindo que o tratamento térmico a $450{ }^{\circ} \mathrm{C}$ por $4,5 \mathrm{~h}$ tenha promovido uma remoção parcial de alumínio da rede cristalina, o que foi confirmado pelo espectro $\mathrm{de}^{27} \mathrm{Al} \mathrm{MAS} / \mathrm{RMN}$ (Figura 3Sa, material suplementar) que indicou a presença de um pico pouco intenso a $0,5 \mathrm{ppm}$, correspondente a espécies de alumínio extrarrede (ALER). Quanto à amostra TZSM5 , os resultados sugerem que o tratamento térmico sob condições severas $\left(1000{ }^{\circ} \mathrm{C}\right.$, por $\left.2 \mathrm{~h}\right)$ acarretou uma intensa desaluminização da rede cristalina, com formação de espécies de alumínio extrarrede (ALER) em diferentes coordenações, como constatado no espectro de MAS/RMN do ${ }^{27} \mathrm{Al}$ (Figura 3Sc, material suplementar). No espectro de ${ }^{29} \mathrm{Si} \mathrm{MAS} / \mathrm{RMN}$, o pico correspondente às espécies 3Si-1Al não pode ser identificado, o que impossibilitou a estimativa da razão $\mathrm{Si} /$
$\mathrm{Al}$ por esta técnica. A presença das espécies de ALER no interior da estrutura porosa da TZSM-5 impediria o acesso tanto aos microporos existentes na amostra original, quanto aos mesoporos gerados no tratamento térmico. Ainda assim, a comparação entre a área de mesoporos da amostra TZSM-5 e a da amostra original HZSM-5 mostra um aumento da área de mesoporos significativo, um resultado similar ao apresentado por Zhang e colaboradores ${ }^{9}$ ao estudarem a geração de mesoporos em zeólita HZSM-5 por tratamento térmico em condições similares. A amostra submetida ao tratamento básico (BZSM-5) apresentou razão $\mathrm{Si} / \mathrm{Al}$ da rede cristalina inferior à da amostra HZSM-5, numa tendência consistente com a dessilicação proporcionada por este procedimento. A presença de espécies de ALER em quantidades que não podem ser ignoradas (pico a 0,5 ppm no espectro de MAS/RMN do ${ }^{27} \mathrm{Al}$ mostrado na Figura 3Sb, material suplementar) indica que a dessilicação foi acompanhada da remoção de alumínio estrutural. Resultados similares foram relatados por outros autores ${ }^{13,15}$ ao investigarem a dessilicação da ZSM-5 por tratamentos em meio alcalino. De acordo com Gil et al., ${ }^{13}$ a formação das espécies de alumínio extrarrede seria responsável pela gradativa amorfização da estrutura observada com o aumento da concentração de $\mathrm{NaOH}$ empregada no tratamento básico. Por outro lado, Song et $a l .{ }^{15}$ procuraram explicar a remoção do alumínio juntamente com a do silício pelo tratamento alcalino. Segundo estes autores, a remoção de átomos de silício estruturais tornaria menos estáveis as ligações envolvendo os átomos de alumínio da rede localizados nas proximidades, tornando mais fácil a sua remoção.

Os dados de cristalinidade apresentados na Tabela 2 indicam que os tratamentos realizados reduziram a cristalinidade das amostras, sendo este efeito mais importante no caso do tratamento térmico. No entanto, como observado a partir dos difratogramas de raios $\mathrm{X}$ mostrados na Figura 2, apesar das alterações observadas nas propriedades texturais e na composição química da rede cristalina após os tratamentos térmico e básico, a estrutura cristalina das zeólitas tratadas foi preservada. Este fato confirma a elevada estabilidade térmica e química das zeólitas pentasil, dentre as quais se destaca a ZSM-5, em função das suas altas relações atômicas Si/Al (maiores do que 15).

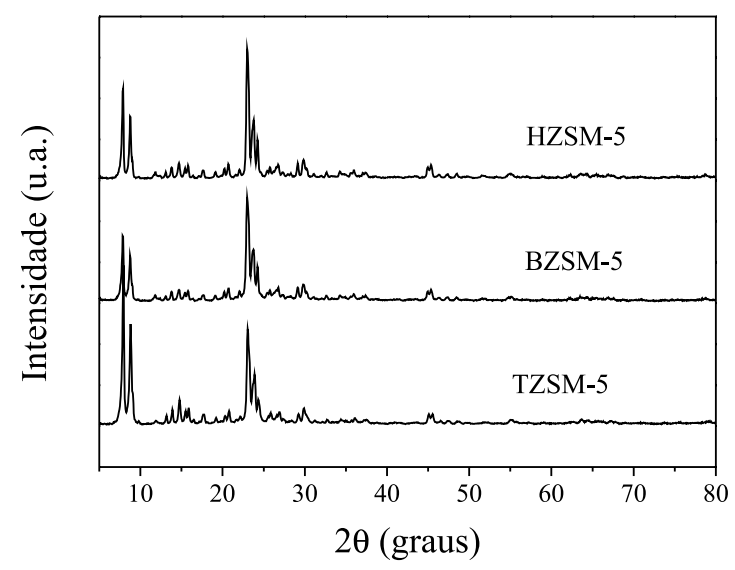

Figura 2. Difratogramas de raios X das amostras HZSM-5, TZSM-5 e BZSM-5

As propriedades ácidas das amostras HZSM-5, TZSM-5 e BZSM-5 foram determinadas através da dessorção de $\mathrm{NH}_{3}$ à temperatura programada. Os perfis de dessorção de $\mathrm{NH}_{3}$ são mostrados na Figura 3, sendo que o perfil da amostra TZSM-5 foi representado separadamente devido à pequena adsorção de $\mathrm{NH}_{3}$ apresentada por esta amostra. Com o objetivo de quantificar a distribuição de força ácida dos sítios, os perfis foram decompostos em três picos apresentando temperatura máxima de dessorção nas faixas entre 260-280, 
Tabela 3. Densidade de sítios ácidos presente nas amostras HZSM-5, TZSM-5 e BZSM-5

\begin{tabular}{|c|c|c|c|c|c|c|c|}
\hline \multirow{2}{*}{ Amostra } & \multirow{2}{*}{$\begin{array}{l}\text { Densidade total de sítios } \\
\text { ácidos }\left(\mu \mathrm{mol} \mathrm{NH}_{3} \mathrm{~g}^{-1}\right)\end{array}$} & \multicolumn{2}{|c|}{ Sítios fracos } & \multicolumn{2}{|c|}{ Sítios intermediários } & \multicolumn{2}{|c|}{ Sítios fortes } \\
\hline & & $\mathrm{T}\left({ }^{\circ} \mathrm{C}\right)$ & $\%$ & $\mathrm{~T}\left({ }^{\circ} \mathrm{C}\right)$ & $\%$ & $\mathrm{~T}\left({ }^{\circ} \mathrm{C}\right)$ & $\%$ \\
\hline HZSM-5 & 1627 & 288 & 41,5 & 345 & 13,4 & 473 & 45,1 \\
\hline BZSM-5 & 1339 & 288 & 40,0 & 345 & 23,6 & 476 & 36,4 \\
\hline TZSM-5 & 52 & 269 & 49 & 333 & 51 & - & - \\
\hline
\end{tabular}

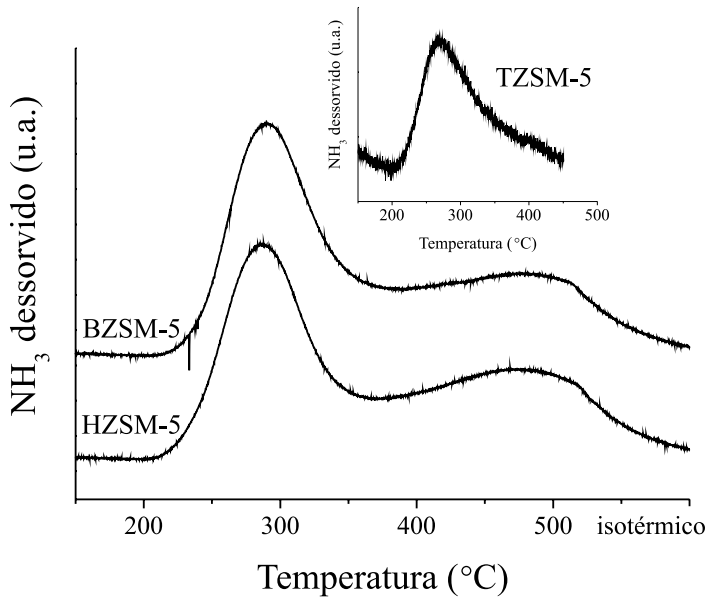

Figura 3. Perfis de dessorção de $\mathrm{NH}_{3}$ à temperatura programada para as amostras HZSM-5, TZSM-5 e BZSM-5

330-350 e 470-480 ${ }^{\circ} \mathrm{C}$, correspondendo aos sítios ácidos fracos, intermediários e fortes, respectivamente. Os resultados são apresentados na Tabela 3 juntamente com os valores de densidade total de sítios ácidos, calculados a partir dos teores de $\mathrm{NH}_{3}$ quimissorvido. A decomposição dos perfis de dessorção à temperatura programada de $\mathrm{NH}_{3}$ é mostrada na Figura $4 \mathrm{~S}$, material suplementar.

Os perfis para as amostras HZSM-5 e BZSM-5 são muito semelhantes, tendo sido observada a presença dos ácidos fracos, intermediários e fortes. As temperaturas referentes aos máximos de dessorção de cada pico foram virtualmente idênticas, indicando que o tratamento básico não teve efeito importante sobre a força dos sítios ácidos, muito embora tenha afetado a quantidade relativa de sítios intermediários e fortes. Como pode ser constatado a partir dos resultados mostrados na Tabela 3 , o tratamento básico provocou uma ligeira redução na densidade total de sítios, que foi acompanhada de um aumento na fração de sítios de força intermediária às expensas dos sítios ácidos fortes. Não houve efeito importante no percentual de sítios fracos. Por outro lado, o tratamento térmico reduziu drasticamente a densidade total de sítios ácidos, além de ter eliminado totalmente os sítios ácidos fortes.

As amostras HZSM-5, TZSM-5 e BZSM-5 foram usadas como catalisadores para a conversão de etanol em hidrocarbonetos, especialmente olefinas como o propeno. Os testes catalíticos foram realizados a $500{ }^{\circ} \mathrm{C}$ e os resultados mostraram que o etanol foi totalmente consumido na presença dos três catalisadores testados. A conversão de etanol não se alterou ao longo do tempo de reação (265 min). Os produtos formados foram eteno $\left(\mathrm{C}_{2}{ }^{ }\right)$, propeno $\left(\mathrm{C}_{3}{ }^{ }\right)$, buteno $\left(\mathrm{C}_{4}{ }^{ }\right)$, uma fração $\mathrm{C}_{5}\left(\mathrm{C}_{5}+\mathrm{C}_{5}{ }^{=}\right)$, uma fração de compostos com mais de seis átomos de carbono $\left(\mathrm{C}_{6}{ }^{+}\right.$aromáticos $)$e uma fração de parafinas $\mathrm{C}_{1}$ a $\mathrm{C}_{4}$. Não foi observada a formação de éter etílico.

A conversão do etanol em hidrocarbonetos mais pesados é um processo complexo que envolve diversas reações catalisadas por sítios ácidos. Inicialmente, de acordo com a literatura, ${ }^{16}$ ocorre a desidratação do etanol a eteno, cuja primeira etapa é a adsorção do etanol em sítios ácidos de Brönsted com a formação de um íon oxônio. Em seguida, forma-se um grupo etoxi com a eliminação de uma molécula de água; este grupo libera eteno e outra molécula de água, restaurando o sítio de Brönsted. Deve-se ressaltar, também, que a altas temperaturas o eteno pode ser um produto secundário de craqueamento térmico. ${ }^{4}$

A produção de hidrocarbonetos mais pesados a partir do eteno ocorre através de etapas de oligomerização, cujos intermediários são carbocátions que são convertidos através de reações de craqueamento, isomerização, ciclização, aromatização e transferência de hidrogênio. ${ }^{17}$ Nestas reações, as condições experimentais usadas e a competição entre acidez e seletividade de forma do catalisador desempenham papéis importantes. ${ }^{7}$

A Tabela 4 apresenta a distribuição dos produtos de reação após 10 min. Os tratamentos realizados na amostra HZSM-5 acarretaram modificações importantes na distribuição dos produtos formados. Observa-se que os principais produtos formados foram eteno e propeno. A amostra HZSM-5 apresentou uma razão molar propeno/ eteno de 0,46 e uma formação de olefinas $\mathrm{C}_{2}-\mathrm{C}_{4}$ de 77,3\%. Após o tratamento básico com uma solução $0,2 \mathrm{~mol} \mathrm{~L}^{-1}$ de $\mathrm{NaOH}$ a $85^{\circ} \mathrm{C}$ por $2 \mathrm{~h}$, a amostra BZSM-5 mostrou uma razão molar propeno/eteno de 0,53 e uma formação de olefinas $\mathrm{C}_{2}-\mathrm{C}_{4}$ de $82,3 \%$, indicando que este tratamento favoreceu a formação de olefinas e aumentou a proporção de propeno obtido. Também foi notado um aumento da quantidade da fração $\mathrm{C}_{5}+\mathrm{C}_{5}=$ e um decréscimo na formação de parafinas $\left(\mathrm{C}_{1}-\mathrm{C}_{4}\right) \mathrm{e}$ de compostos com mais de seis átomos de carbono $\left(\mathrm{C}_{6}{ }^{+}\right.$aromáticos $)$. A diminuição da quantidade de parafinas e olefinas observada para a amostra BZSM-5 está relacionada com o decréscimo na quantidade de sítios ácidos fortes deste material, visto que estes sítios catalisam as reações de transferência de hidrogênio que transformam olefinas em parafinas. ${ }^{4}$ Por outro lado, na amostra submetida ao tratamento térmico a $1000{ }^{\circ} \mathrm{C}$ por $2 \mathrm{~h}$ (TZSM-5), o eteno foi o único produto formado em quantidades significativas, indicando que a etapa inicial da reação (desidratação do etanol a eteno) é promovida pelos sítios ácidos de menor força.

Tabela 4. Distribuição dos produtos de reação (\% molar) após 10 min de reação. Conversão $100 \% ; \mathrm{T}=500{ }^{\circ} \mathrm{C} ; \mathrm{WHSV}=6,5 \mathrm{~g}_{\mathrm{EtOH}} \mathrm{h}^{-1} \mathrm{~g}_{\text {cat }}{ }^{-1}$

\begin{tabular}{cccc}
\hline Amostra & HZSM-5 & BZSM-5 & TZSM-5 \\
\hline $\mathrm{C}_{2}{ }^{=}$ & 48,00 & 48,29 & 99,36 \\
$\mathrm{C}_{3}{ }^{=}$ & 22,30 & 25,74 & 0,04 \\
$\mathrm{C}_{4}{ }^{-}$ & 7,01 & 8,29 & 0,06 \\
$\mathrm{C}_{5}+\mathrm{C}_{5}{ }^{=}$ & 1,75 & 2,25 & 0,00 \\
$\mathrm{C}_{6}{ }^{+}$ & 6,77 & 5,47 & 0,33 \\
$\mathrm{C}_{1}-\mathrm{C}_{4}$ & 14,17 & 9,96 & 0,07 \\
\hline
\end{tabular}

A investigação dos efeitos do tratamento básico sobre as propriedades físico-químicas e a distribuição dos produtos de reação indica que tanto as propriedades texturais quanto a acidez foram significativamente alteradas. Com relação ao tratamento térmico, a propriedade que mostrou mudanças mais significativas foi a acidez, cujo decréscimo foi muito grande. De acordo com a literatura, a 
diminuição da acidez do material atenua as etapas de transformação do eteno em hidrocarbonetos mais pesados. ${ }^{18,19}$

O comportamento da seletividade aos produtos ao longo da reação é mostrado nas Figuras 4 a 6 para as três amostras testadas. Para as amostras HZSM-5 e TZSM-5 a seletividade aos produtos permaneceu praticamente constante ao longo do tempo de reação, não sendo observada desativação destes materiais.

A Figura 5 mostra que para a zeólita BZSM-5 a seletividade ao eteno aumentou ao longo do tempo de reação, enquanto que a

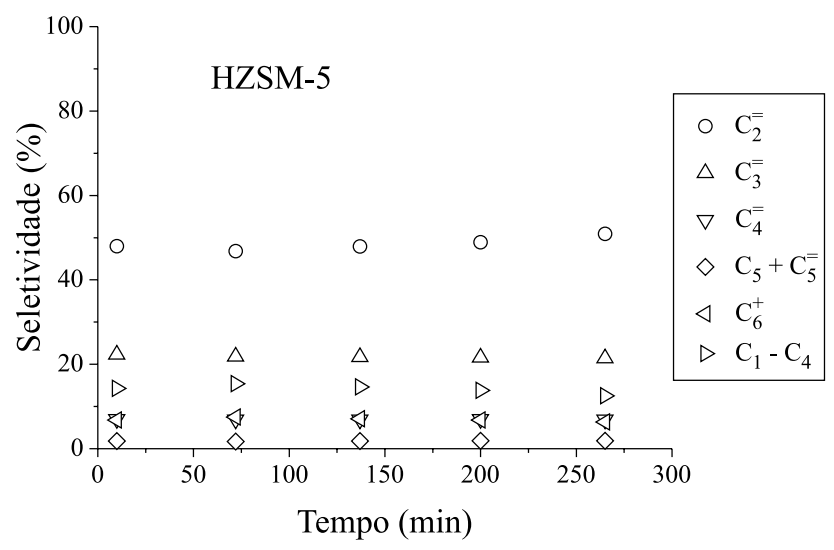

Figura 4. Variação da seletividade aos produtos ao longo do tempo de reação para a amostra HZSM-5. Conversão $100 \% ; \mathrm{T}=500{ }^{\circ} \mathrm{C}$; WHSV = $6,5 g_{\text {EtoH }} h^{-1} g_{\text {cat }}{ }^{-1}$

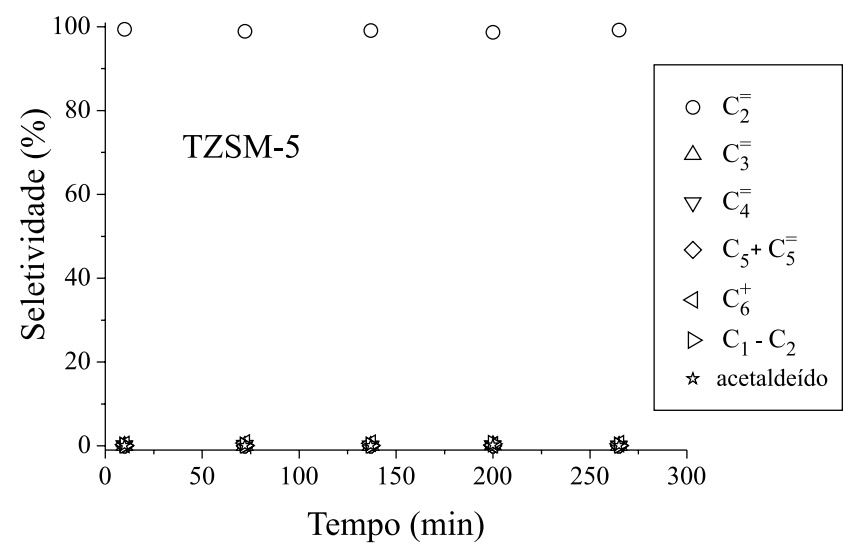

Figura 5. Variação da seletividade aos produtos ao longo do tempo de reação para a amostra TZSM-5. Conversão $100 \% ; \mathrm{T}=500{ }^{\circ} \mathrm{C} ; \mathrm{WHSV}=$ $6,5 g_{\text {EtOH }} h^{-1} g_{\text {cat }}^{-1}$

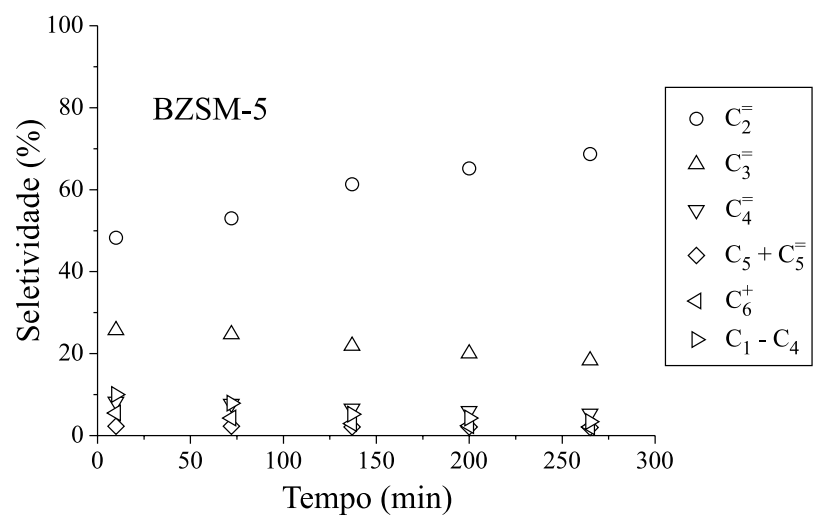

Figura 6. Variação da seletividade aos produtos ao longo do tempo de reação para a amostra BZSM-5. Conversão $100 \% ; \mathrm{T}=500{ }^{\circ} \mathrm{C} ; \mathrm{WHSV}=$ $6,5 g_{\text {EtOH }} h^{-1} g_{\text {cat }}{ }^{-1}$ seletividade ao propeno, buteno, compostos $\mathrm{C}_{6}{ }^{+}$e parafinas $\mathrm{C}_{1}-\mathrm{C}_{4}$ diminuiu. Tal comportamento pode estar associado ao aumento da mesoporosidade proporcionado pelo tratamento básico, favorecendo a formação de coque por aumentar o espaço disponível para acomodar as moléculas volumosas do mesmo. A formação de coque desativaria preferencialmente os sítios de maior força (fortes e intermediários), justificando o gradativo favorecimento à formação do eteno, que ocorre nos sítios de menor força ácida.

\section{CONCLUSÕES}

Os tratamentos estudados (térmico e básico) foram eficazes para a geração de mesoporosidade. $\mathrm{O}$ tratamento térmico acarretou uma importante desaluminização da amostra que proporcionou um pequeno aumento na área e volume de mesoporos, a formação de espécies de alumínio extrarrede e uma drástica redução da densidade de sítios ácidos. Já o tratamento básico, responsável pela dessilicação da amostra, possibilitou um aumento significativo dos mesoporos e um ligeiro decréscimo da densidade de sítios ácidos.

Com relação ao desempenho dos catalisadores zeolíticos preparados frente à conversão do etanol, os tratamentos alteraram significativamente a distribuição dos produtos formados e a estabilidade dos catalisadores. A formação de olefinas e o aumento da proporção de propeno formado no início da reação foram verificados para a amostra preparada pelo tratamento básico, enquanto que o tratamento térmico proporcionou apenas a formação de eteno. As propriedades ácidas tiveram uma forte influência sobre o desempenho catalítico das amostras sintetizadas. Com respeito à estabilidade, o tratamento básico gerou uma amostra cuja seletividade aos produtos foi alterada durante o tempo de reação, o que pode estar relacionado com o grande aumento do volume de mesoporos favorecendo a formação de coque.

\section{MATERIAL SUPLEMENTAR}

As Figuras 1S a 4S mencionadas ao longo do texto encontram-se disponíveis gratuitamente, sob a forma de arquivo pdf, em http:// quimicanova.sbq.org.br.

\section{AGRADECIMENTOS}

Ao CENPES/PETROBRAS pelo apoio financeiro. Ao Dr. R. C. P. Bittencourt (CENPES/PETROBRAS) pela valiosa colaboração no preparo e caracterização das amostras. À S. M. C. Menezes e N. M. S. Ruiz (CENPES/PETROBRAS) pela realização das análises por MAS/RMN de ${ }^{29} \mathrm{Si} \mathrm{e}^{27} \mathrm{Al}$. C. A. Henriques agradece ao CNPq e ao Programa PROCIÊNCIA da UERJ o apoio financeiro. F. M. Z. Zotin agradece ao Programa PROCIÊNCIA da UERJ.

\section{REFERÊNCIAS}

1. Song, Z.; Takahashi, A.; Mimura, N.; Fujitani, T.; Catal. Lett. 2009, 131, 364.

2. Xia, W.; Takahashi, A.; Nakamura, I.; Shimada, H.; Fujitani, T.; J. Mol. Catal. A: Chem. 2010, 328, 114.

3. Makarfi, Y.; Yakimova, M.; Lermontov, A.; Erofeev, V.; Koval, L.; Tretiyakov, V.; Chem. Eng. J. 2009, 154, 396.

4. Song, Z.; Takahashi, A.; Nakamura, I.; Fujitani, T.; Appl. Catal., A 2010, 384, 201.

5. Gayubo, A.; Alonso, A.; Valle, B.; Aguayo, A.; Bilbao, J.; Ind. Eng. Chem. Res. 2010, 49, 10836.

6. Ferreira Madeira, F.; Gnep, N.; Magnoux, P.; Vezin, H.; Maury, S.; Cadran, N.; Chem. Eng J. 2010, 161, 403. 
7. Gayubo, A.; Alonso, A.; Valle, B.; Aguayo, A.; Bilbao, J.; Appl. Catal., B 2010, 97, 299.

8. Christensen, C.; Johannsen, K.; Tornqvist, E.; Schmidt, I.; Topsoe, H.; Christensen, C.; Catal. Today 2007, 128, 117.

9. Zhang, C.; Liu, Q.; Xu, Z.; Wan, K.; Microporous Mesoporous Mater. 2003, 62, 157 .

10. Groen, J.; Moulijn, J.; Ramírez, J.; Microporous Mesoporous Mater. $\mathbf{2 0 0 5}, 87,153$

11. Ogura, M.; Shinomiya, S.; Tateno, J.; Nara, Y.; Nomura, M.; Kikuchi, E.; Matsukata, M.; Appl. Catal., A 2001, 219, 33.

12. Nascimento, T. L. P. M.; Dissertação de Mestrado, Universidade do Estado do Rio de Janeiro, Brasil, 2010.

13. Gil, B.; Mokrzycki, L.; Sulikowski, B.; Olejniczak, Z.; Walas, S.; Catal. Today 2010, 152, 24.
14. Menezes, S. M. C. ; Camorim, V. L. ; Lam, Y. L. ; SanGil, R. A. S. ; Bailly, A. ; Amoureux, J. P.; Appl. Catal., A 2001, 207, 367.

15. Song, Y.-Q.; Feng, Y-L.; Liu, F.; Kang, C-L.; Zhou, X-L.; Xy, L-Y.; Yu, G-X.; J. Mol. Catal. A: Chem. 2009, 310, 130.

16. Kondo, J. N.; Ito, K.; Yoda, E.; Wakabayashi, F.; Domen, K.; J. Phys. Chem. B 2005, 109, 10969.

17. Borges, P.; Ramos Pinto, R.; Lemos, M.; Lemos, F.; Vedrine, J. C.; Derouane, E. G.; Ramoa Ribeiro, F.; Appl. Catal., A 2007, 324, 20.

18. Inoue, K.; Okabe, K.; Inaba, M.; Takahara, I.; Murata, K.; React. Kinet. Mech. Catal. 2010, 101, 477.

19. Ferreira Madeira, F.; Gnep N.; Magnoux, P.; Maury, S.; Cadran, N.; Appl. Catal., A 2009, 367, 39. 


\section{GERAÇÃO DE MESOPOROS EM ZEÓLITAS ZSM-5 E SEUS EFEITOS NA CONVERSÃO DO ETANOL EM OLEFINAS}

Ingryd Carvalho Alves, Tâmara Lucia Pacheco de Mattos do Nascimento", Cláudia de Oliveira Veloso, Fátima Maria Zanon Zotin e Cristiane Assumpção Henriques*

Instituto de Química, Universidade do Estado do Rio de Janeiro, Rua São Francisco Xavier, 524, 20550-900 Rio de Janeiro - RJ, Brasil
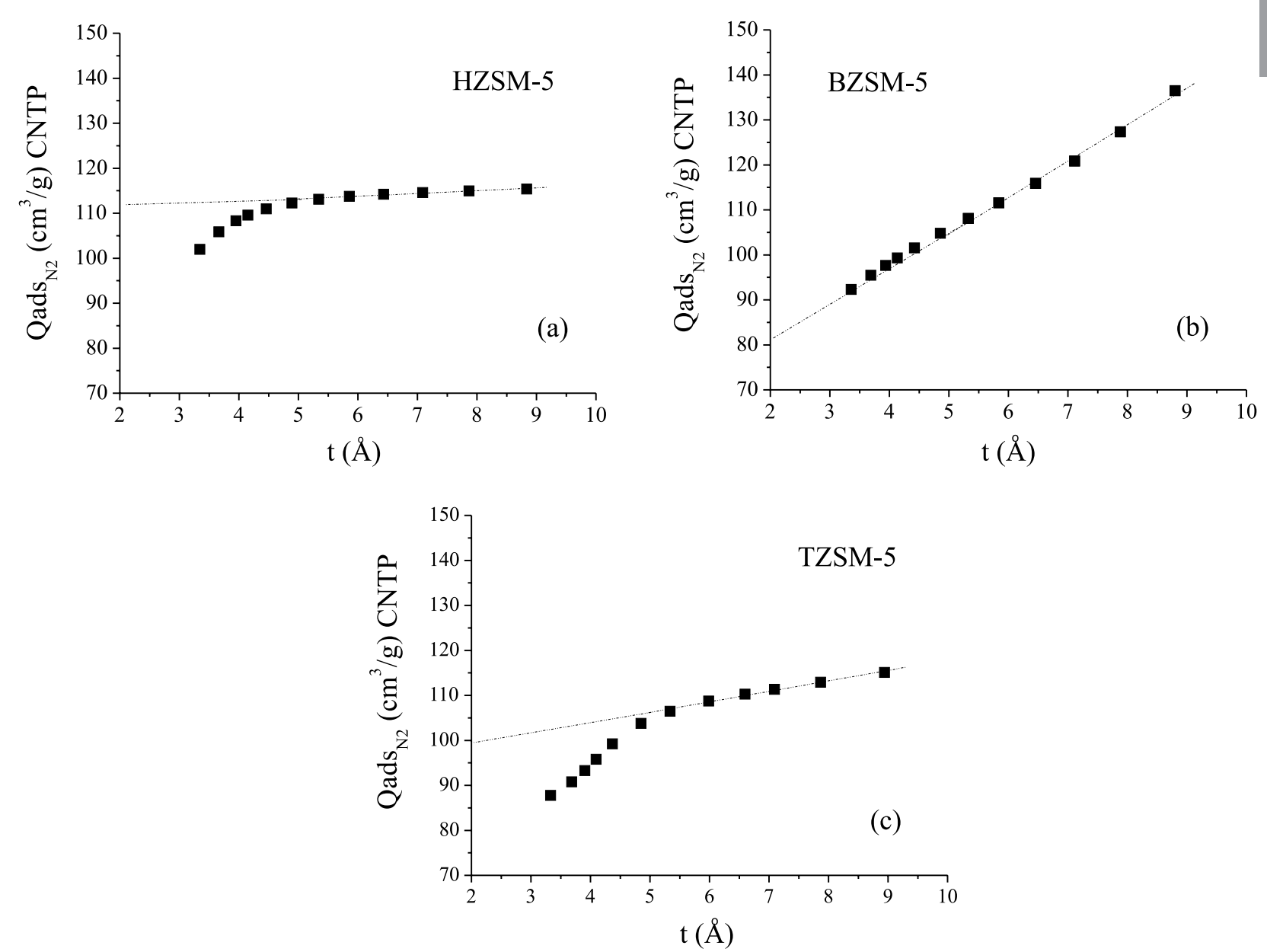

Figura 1S. Isotermas t para as amostras estudadas: (a) HZSM-5; (b) BZSM-5; (c) TZSM-5. A linha pontilhada indica os pontos linearizados (espessura da camada t entre 5 e $9 \AA$ ) para o cálculo do volume de microporos 

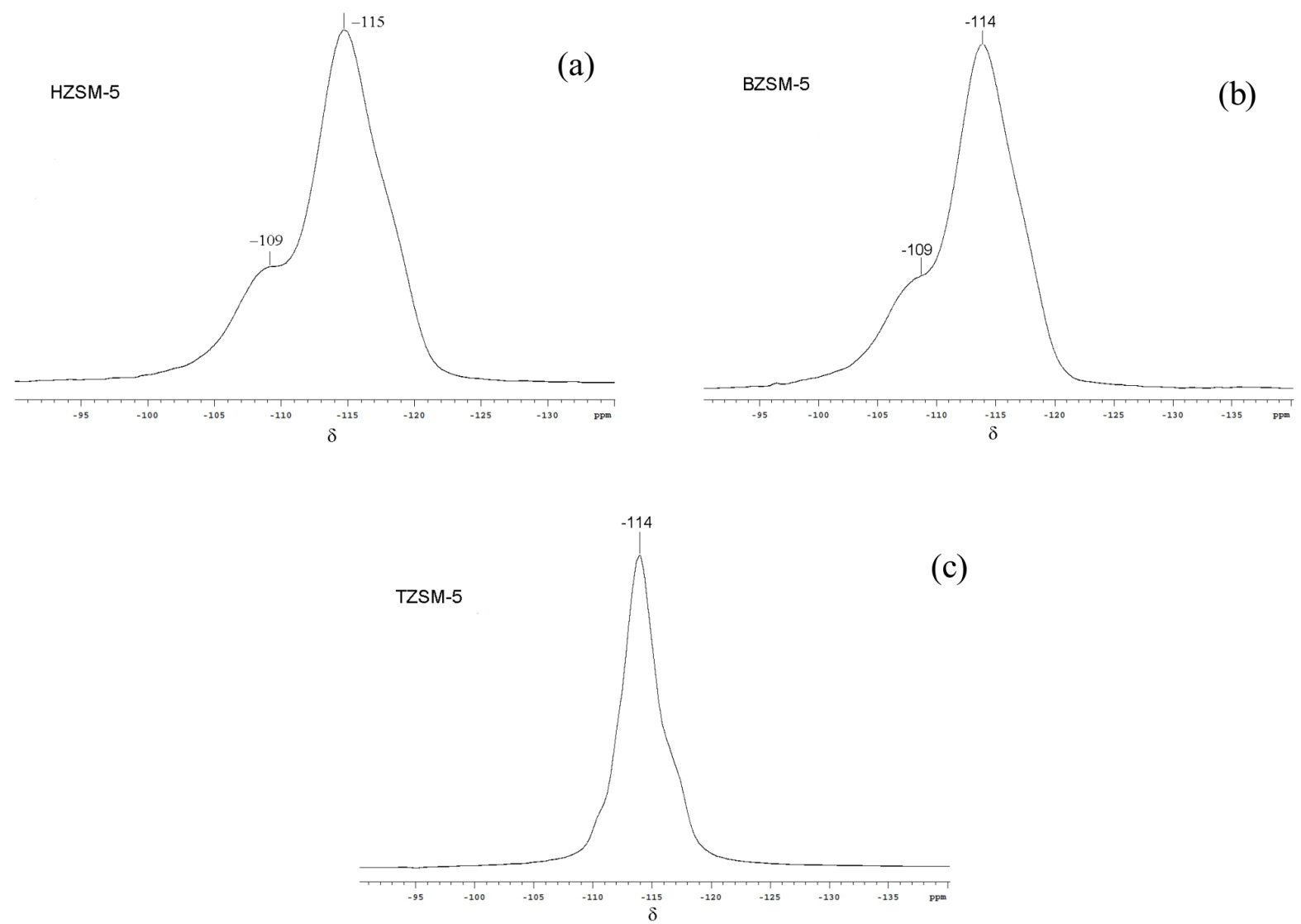

(c)

Figura 2S. Espectros de ${ }^{29}$ Si MAS/RMN das amostras estudadas. (a) HZSM-5; (b) BZSM-5; (c) TZSM-5. Deslocamento químico: 4Si-0Al $\delta \approx$ - 114 ppm; 3Si$1 \mathrm{Al} \delta \approx-109 \mathrm{ppm}$
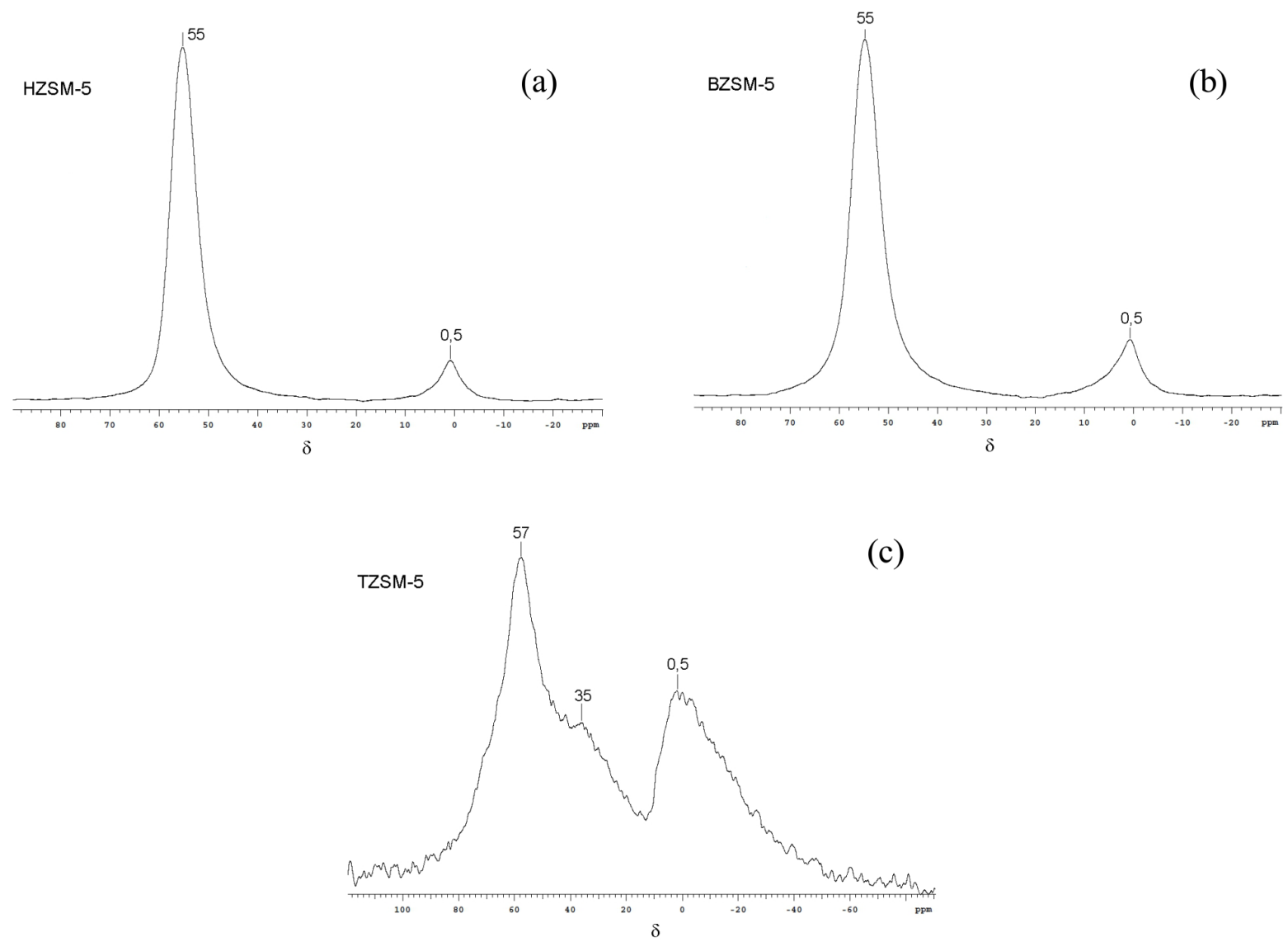

Figura 3S. Espectros de ${ }^{27}$ Al MAS/RMN das amostras estudadas. (a) HZSM-5; (b) BZSM-5; (c) TZSM-5. Deslocamento químico: Al tetraédrico $\delta \approx 55$ ppm; Al octaédrico $\delta \approx 0,5 \mathrm{ppm}$ 

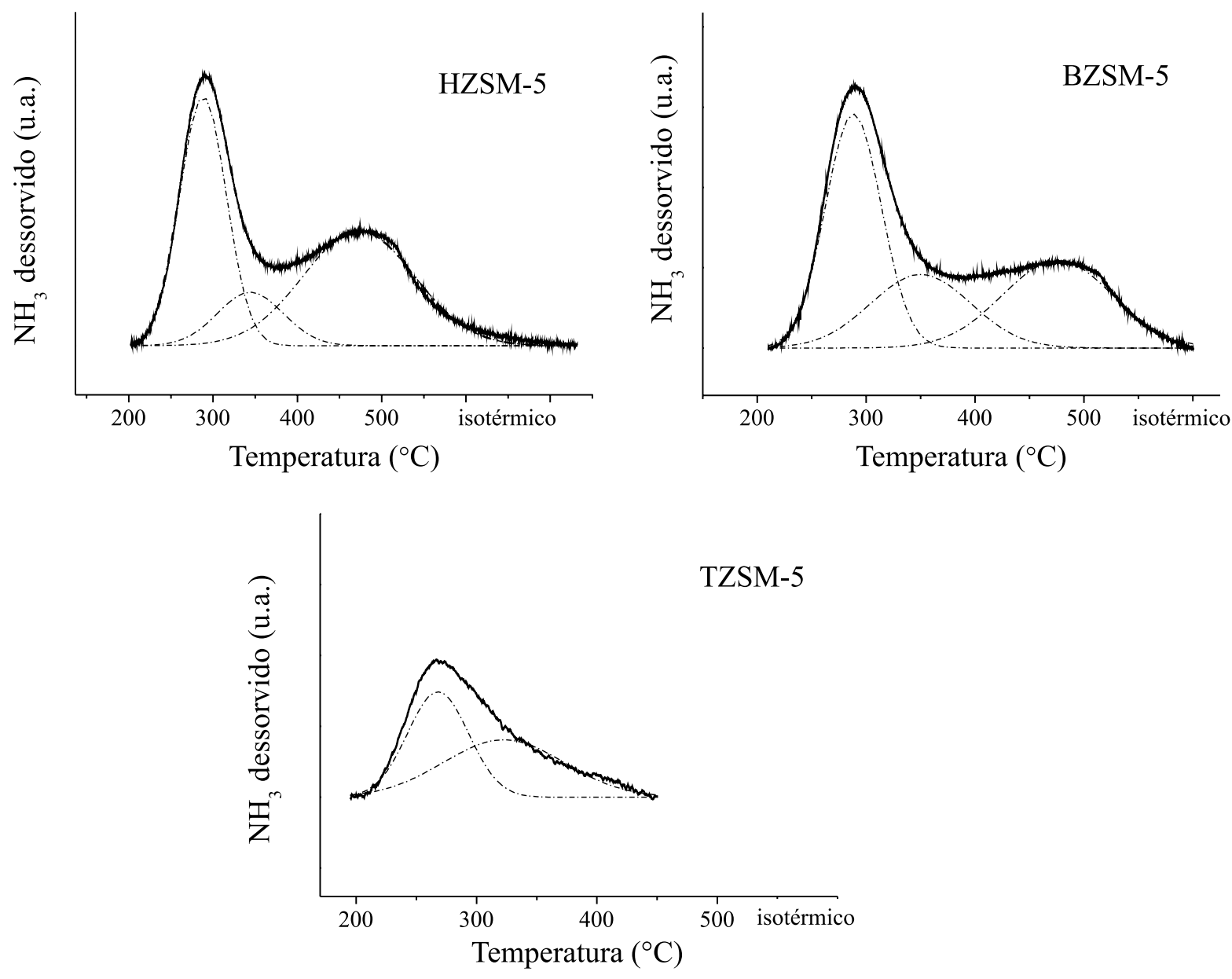

Figura 4S. Decomposição dos perfis de TPD de $\mathrm{NH}_{3}$. 